\title{
Detecting Dark Matter in Exoplanets
}

\section{Measuring the temperatures of massive exoplanets could reveal the effect of dark matter, potentially allowing researchers to confirm the galactic distribution of this mysterious substance.}

\author{
By Marric Stephens
}

F or dark matter detectors, bigger is usually better, as that means that the detector can potentially interact with more dark matter particles. But even the largest are tiny compared to a newly proposed detector [1]. Rebecca Leane of the SLAC National Accelerator Laboratory at Stanford University and Juri Smirnov of Ohio State University, Columbus, suggest searching for dark matter by measuring its effect on the temperatures of exoplanets and brown dwarfs up to 55 times the mass of Jupiter. With tens of thousands of suitable exoplanets expected to be found in the next five years, these measurements could detect-or rule out-dark matter particles with masses greater than that of an electron.

The prevailing model of dark matter's distribution in the Universe has our Galaxy sitting within a spherical dark matter "halo," whose density increases toward the Galactic Center. Occasionally, a dark matter particle within this halo may scatter from an electron or a nucleon in an astronomical body before



Credit: Artsiom P/stock.adobe.com becoming trapped by the body's gravity. Some hypothesized forms of dark matter annihilate to produce photons and other particles, meaning that they might heat a body if a significant quantity of dark matter particles accumulate.

Leane and Smirnov show that for gas giants orbiting stars local to Earth, this heating could be measurable using NASA's soon-to-be-launched James Webb Space Telescope. The telescope might also detect the signal in more distant brown dwarfs and "rogue" exoplanets (planets that lack a parent star), with those closest to the Galactic Center predicted to exhibit the greatest dark matter capture rates and the highest temperatures. As well as providing information on the nature of dark matter, these measurements could reveal parameters of the dark matter halo, such as its density profile.

Marric Stephens is a Corresponding Editor for Physics based in Bristol, UK.

\section{REFERENCES}

1. R. K. Leane and J. Smirnov, "Exoplanets as sub-GeV dark matter detectors," Phys. Rev. Lett. 126, 161101 (2021). 